\title{
Design of two-channel IIR filter banks with arbitrary group delay
}

\author{
J.-H.Lee and I.-C.Niu
}

\begin{abstract}
The nonlinear optimisation problem that results from considering the design of a twochannel nonuniform division filter bank is solved. This is through a frequency sampling and iterative approximation technique to find the tap coefficients and the reflection coefficients for the numerator and the denominator of the IIR analysis filters. An efficient stabilisation procedure ensures that the reflection coefficients lie in $(-1,1)$. Simulation examples are provided for illustration.
\end{abstract}

\section{Introduction}

Quadrature mirror filter (QMF) banks find applications in sub-band coding of speech signals [1], communication systems [2], short-time spectral analysis [3], and subband coding of image signals [4]. In these applications, a QMF bank is used to decompose a signal into sub-bands with equal bandwidth, and the sub-band signals in the analysis system are decimated by an integer which is equal to the number of the sub-bands. However, uniform subband decomposition is not an appropriate scheme to match the requirements for the sub-band coding of speech and audio signals. The most appropriate decomposition must consider the critical bands of the ear. It has been mentioned previously [5] that these critical bands have nonuniform bandwidths and cannot be easily constructed by a conventional tree structure based on two-channel QMF banks. Therefore, it is worth exploiting the design problem of twochannel nonuniform division filter (NDF) banks.

The conditions on the analysis and synthesis filters of NDF banks, as well as methods to design them, are presented elsewhere [5]. Owing to the difficulty in solving the design problem with nonlinear constraints, a structure and design procedure for pseudo-QMF banks has been presented [6]. The main drawback of this method is that FIR filters with complex coefficients are required by the resulting NDF bank to reduce the aliasing distortion. A structure for two-channel NDF banks has been considered [7], and design methods have been proposed [8] for optimally designing NDF banks based on $L_{1}$ and $L_{2}$ error criteria.

Designing an NDF bank has been widely considered [5-8]. However, linear-phase FIR analysis filters were employed for developing these design techniques.

(C) IEE, 2000

IEE Proceedings online no. 20000489

DOI: 10.1049 /ip-vis:20000489

Paper first received 19 May 1999 and in final revised form 28th March 2000

The authors are with the The Department of Electrical Engineering, National Taiwan University, Taipei, 106 Taiwan
Although using linear-phase FIR analysis filters leads to a favorable problem formulation for design, the system delay $k_{d}$ of the designed NDF bank is determined by the lengths of FIR filters used; hence, the long overall system delay of an NDF bank with linear-phase FIR analysis filters may prohibit practical applications. It is well known that an IIR filter requires lower order than an FIR filter under the same stopband energy. For a given passband group delay, IIR NDFs have better passband ripple/stopband attenuation and/or lower computational complexity than FIR NDFs under the same stopband energy for the analysis and synthesis filters. Several approaches dealing with the design problem of two-channel IIR uniform division filter banks with conventional filter structures have been reported [9-11]. There is, however, practically no published work concerning the design of two-channel IIR NDF banks with arbitrary group delay.

In this paper, we consider the design of two-channel IIR NDF banks with arbitrary group delay in the sense of $L_{2}$ error criteria. Utilising a lattice structure for the denominators of the IIR analysis filters, a design technique based on an approximation scheme is presented for efficiently solving the resulting design problem that is basically a nonlinear optimisation problem. During the design process, the proposed technique finds the tap coefficients for the numerators and the reflection coefficients for the denominators of the IIR analysis filters simultaneously. It ensures the stability of the designed IIR analysis filters by incorporating an efficient stabilisation procedure to make the magnitude of each reflection coefficient within -1 and +1 . Computer simulations show that the IIR NDF banks designed using the proposed technique provide a satisfactory performance, although the designed results are only local solutions (instead of the global optimum).

\section{Two-channel NDF banks with arbitrary group delay}

Consider the two-channel NDF bank, with the architecture given previously [7], which is shown in Fig. 1. The analysis low-pass and high-pass filters are designated by $H_{0}(z)$ and $H_{1}(z)$, respectively, and the synthesis lowpass and high-pass filters are designated by $F_{0}(z)$ and $F_{1}(z)$, respectively. $B_{0}(z)$ and $B_{1}(z)$ are low-pass filters

IEE Proc.-Vis. Image Signal Process., Vol. 147, No. 6, December 2000 


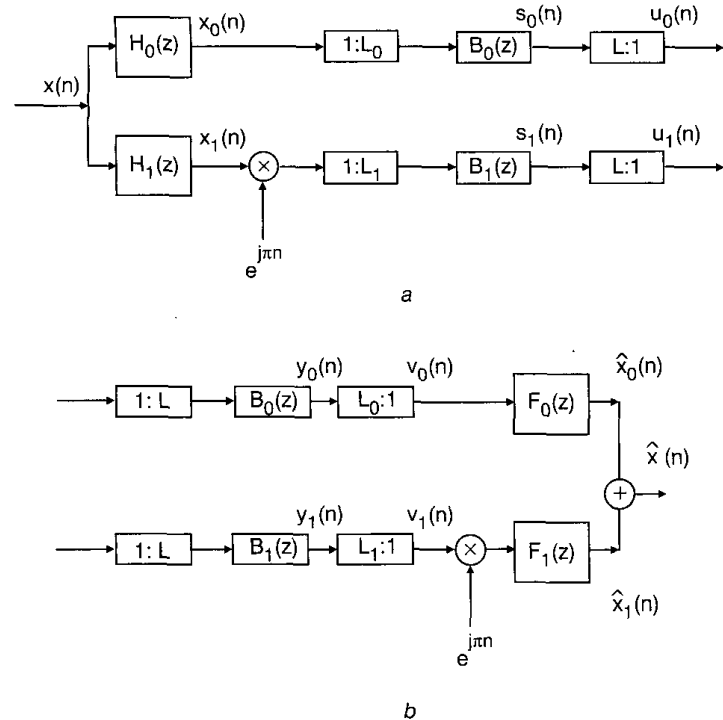

Fig. 1 Two-channel NDF bank system structure

a Analysis system

$b$ Synthesis system

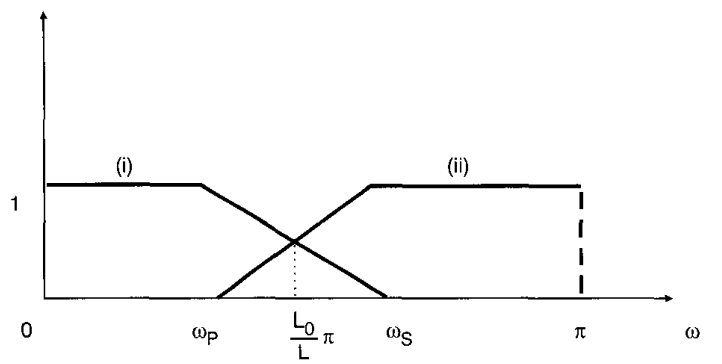

Fig. 2 Desired magnitude responses for analysis filters

(i) $\left|H_{0}\left(e^{j t(t)}\right)\right| \sqrt{ }\left(L_{0} L\right)$

(ii) $\left|H_{1}\left(e^{j \omega}\right)\right| / \sqrt{ }\left(L_{1} L\right)$

responsible for achieving aliasing-free operation during the rational decimation and interpolation. It can be shown that using the modulations of multiplying $\exp (j n \pi)$ in the highpass sub-band channel leads to the favourable result that $B_{1}(z)$ can be a low-pass filter with real coefficients. The desired magnitude responses for the analysis filters $H_{0}(z)$ and $H_{1}(z)$ with passband widths equal to $L_{0} \pi / L$ and $L_{1} \pi / L$, respectively, are shown in Fig. 2. $L=L_{0}+L_{1}$, and $\omega_{p}$ and $\omega_{s}$ denote the related band-edge frequencies satisfying $\omega_{p}+\omega_{s}=2 \pi L_{0} / L$.

Assume that the associated magnitude responses are set to

$$
\left\{\begin{array}{r}
B_{0}(\omega)=1, \quad \text { for } \omega \in\left[0, \frac{\omega_{s}}{L_{0}}\right] \\
\text { and } B_{0}(\omega)=0, \quad \text { for } \omega \in\left[\frac{2 \pi-\omega_{s}}{L_{0}}, \pi\right], \\
B_{1}(\omega)=1, \quad \text { for } \omega \in\left[0, \frac{\pi-\omega_{p}}{L_{1}}\right] \\
\text { and } B_{1}(\omega)=0, \text { for } \omega \in\left[\frac{\pi+\omega_{p}}{L_{1}}, \pi\right]
\end{array}\right.
$$

Further, assume that $H_{0}(z)$ and $H_{1}(z)$ have zero stopband response. As shown previously [8], the input/output relationship of the NDF bank in the frequency domain is given by

$$
\begin{aligned}
\hat{X}\left(e^{j \omega}\right)= & \frac{e^{-j \omega G_{0}}}{L L_{0}}\left[X\left(e^{j \omega}\right) H_{0}\left(e^{j \omega}\right)+X\left(e^{j \omega} W_{L}^{L_{0}}\right)\right. \\
& \left.\times H_{0}\left(e^{j \omega} W_{L}^{L_{0}}\right)+X\left(e^{j \omega} W_{L}^{-L_{0}}\right) H_{0}\left(e^{j \omega} W_{L}^{-L_{0}}\right)\right] F_{0}\left(e^{j \omega}\right) \\
& +\frac{e^{-j \omega G_{3}}}{L L_{1}}\left[X\left(e^{j \omega}\right) H_{1}\left(e^{j \omega}\right)+X\left(e^{j \omega} W_{L}^{L_{1}}\right) H_{1}\left(e^{j \omega} W_{L}^{L_{1}}\right)\right. \\
& \left.+X\left(e^{j \omega} W_{L}^{-L_{1}}\right) H_{1}\left(e^{j \omega} W_{L}^{-L_{1}}\right)\right] F_{1}\left(e^{j \omega}\right)
\end{aligned}
$$

where $G_{0}$ and $G_{1}$ are the resulting group delays of the upper and lower channels, respectively. $W_{L}=\exp (-j 2 \pi / L)$. Substituting $\quad L=L_{0}+L_{1}, \quad F_{0}\left(e^{j \omega}\right)=H_{0}\left(e^{j \omega}\right) \quad$ and $F_{1}\left(e^{j \omega}\right)=-H_{1}\left(e^{j \omega}\right)$ into eqn. 2 yields

$$
\begin{aligned}
\hat{X}\left(e^{j \omega}\right)= & T\left(e^{j \omega}\right) X\left(e^{j \omega}\right)+V_{1}\left(e^{j \omega}\right) X\left(e^{j \omega} W_{L}^{L_{0}}\right) \\
& +V_{2}\left(e^{j \omega}\right) X\left(e^{j \omega} W_{L}^{L_{1}}\right)
\end{aligned}
$$

where

$$
\begin{aligned}
T\left(e^{j \omega}\right)= & \frac{e^{-j \omega G_{0}}}{L L_{0}} H_{0}^{2}\left(e^{j \omega}\right)-\frac{e^{-j \omega G_{1}}}{L L_{1}} H_{1}^{2}\left(e^{j \omega}\right) \\
V_{1}\left(e^{j \omega}\right)= & \frac{e^{-j \omega G_{0}}}{L L_{0}} H_{0}\left(e^{j \omega}\right) H_{0}\left(e^{j \omega} W_{L}^{L_{0}}\right) \\
& -\frac{e^{-j \omega G_{1}}}{L L_{1}} H_{1}\left(e^{j \omega}\right) H_{1}\left(e^{j \omega} W_{L}^{L_{0}}\right) \\
V_{2}\left(e^{j \omega}\right)= & \frac{e^{-j \omega G_{0}}}{L L_{0}} H_{0}\left(e^{j \omega}\right) H_{0}\left(e^{j \omega} W_{L}^{L_{1}}\right) \\
& -\frac{e^{-j \omega G_{1}}}{L L_{1}} H_{1}\left(e^{j \omega}\right) H_{1}\left(e^{j \omega} W_{L}^{L_{1}}\right)
\end{aligned}
$$

The first term of eqn. 3 represents the response of a linear shift-invariant system $T\left(e^{j \omega}\right)$ with input $X\left(e^{j \omega}\right)$, and the other two terms represent the resulting aliasing distortion. Therefore, perfect reconstruction for a group delay $k_{d}$ requires the following conditions:

PR I: $T\left(e^{j \omega}\right)$ must be equal to $e^{-j k_{d} \omega}$ for all $\omega$.

$P R$ 2: The magnitude $V_{1}(\omega)$ of $V_{1}\left(e^{j \omega}\right)$ must be zero, i.e.

$$
V_{1}(\omega)=0, \text { for all } \omega .
$$

PR 3: The magnitude $V_{2}(\omega)$ of $V_{2}\left(e^{j \omega}\right)$ must be zero, i.e.

$$
V_{2}(\omega)=0, \text { for all } \omega .
$$

For simplicity, assuming $G_{0}=G_{1}$, we can neglect the phase term $e^{-j \omega G_{0}}$ in eqn. 4 and express $T\left(e^{j \omega}\right)$ as

$$
\hat{T}\left(e^{j \omega}\right)=\frac{H_{0}^{2}\left(e^{j \omega}\right)}{L L_{0}}-\frac{H_{1}^{2}\left(e^{j \omega}\right)}{L L_{1}}
$$

Furthermore, we note from eqns. 5 and 6 and $L=L_{0}+L_{1}$ that the aliasing distortion can be eliminated if

$$
\begin{aligned}
\hat{V}\left(e^{j \omega}\right)= & \frac{1}{L L_{0}} H_{0}\left(e^{j\left(\omega-\omega_{p}-\omega_{s}\right)}\right) H_{0}\left(e^{j \omega}\right) \\
& -\frac{i}{L L_{1}} H_{1}\left(e^{j\left(\omega-\omega_{p}-\omega_{s}\right)}\right) H_{1}\left(e^{j \omega}\right)
\end{aligned}
$$


equals zero for $\omega_{p} \leq \omega \leq \omega_{s}$. Following eqns. 7 and 8 , we can reformulate the conditions required for perfect reconstruction as follows:

$\begin{cases}T\left(e^{j \omega}\right)=e^{-j k_{d}(\omega)}, & \text { for } 0 \leq \omega \leq \pi, \\ H_{0}\left(e^{j \omega}\right)=0, & \text { for } \omega_{s} \leq \omega \leq \pi, \\ H_{1}\left(e^{j \omega}\right)=0, & \text { for } 0 \leq \omega \leq \omega_{p}, \\ \frac{1}{L L_{0}} H_{0}\left(e^{j \omega}\right) H_{0}\left(e^{j\left(\omega-\omega_{p}-\omega_{s}\right)}\right)=\frac{1}{L L_{1}} H_{1}\left(e^{j\left(\omega-\omega_{p}-\omega_{s}\right)}\right) H_{1}\left(e^{j \omega}\right), & \text { for } \omega_{p} \leq \omega \leq \omega_{s}\end{cases}$

Therefore, the design problem of the two-channel IIR NDF banks of Fig. 1 is finding such IIR analysis filters, i.e. $H_{0}(z)$ and $H_{1}(z)$, that the conditions listed in eqn. 9 can be approximately met.

\section{Design of two-channel IIR NDF banks in $\mathrm{L}_{2}$ sense}

\subsection{Problem formulation}

Here, we consider the design of the two-channel IIR NDF as shown in Fig. 1. Let the low-pass analysis filter be an IIR filter with order $M_{0} / N_{0}$ (i.e. $M_{0}$ zeros and $N_{0}$ poles) and transfer function $H_{0}(z)=A_{0}(z) / B_{N_{0}}(z)$. The numerator $A_{0}(z)$ is an $M_{0}$ th-order polynomial with tap coefficient vector $\boldsymbol{a}_{0}=\left[a_{00}, a_{01}, \ldots, a_{0 M_{0}}\right]^{T}$; and $B_{N_{0}}(z)$ is an $N_{0}$ thorder FIR lattice filter with reflection coefficient vector $\boldsymbol{k}_{0}=\left[k_{01}, k_{02}, \ldots, k_{0} N_{0}\right]^{T}$, where ${ }^{T}$ denotes the transpose operation. Similarly, let the high-pass analysis filter be an IIR filter with order $M_{1} / N_{1}$ and transfer function $H_{1}(z)=A_{1}(z) / B_{N_{1}}(z)$. The numerator $A_{1}(z)$ is an $M_{1}$ thorder polynomial with tap coefficient vector $\boldsymbol{a}_{1}=\left[a_{10}\right.$, $\left.a_{11}, \ldots, a_{1 M_{1}}\right]^{T}$, and $B_{N_{1}}(z)$ is an $N_{1}$ th-order FIR lattice filter with reflection coefficient vector $k_{1}=\left[k_{11}, k_{12}\right.$, $\left.\ldots, k_{1 N_{1}}\right]^{T}$. Fig. 3 shows the system structure for $B_{N_{0}}(z)$ and $B_{N_{1}}(z)$, which can be obtained from the following recursive formula [12]:

$$
\begin{aligned}
& B_{0}(z)=Q_{0}(z)=1 \\
& B_{n}(z)=B_{n-1}(z)+k_{n} z^{-1} Q_{n-1}(z) \\
& Q_{n}(z)=k_{n} B_{n-1}(z)+z^{-1} Q_{n-1}(z)
\end{aligned}
$$

Hence, the overall design task is to find the required tap and reflection coefficients $\left\{a_{i m}, k_{i n}\right\}$ for the stable IIR filters $H_{i}(z), i=0,1$, such that the conditions shown in eqn. 9 must be satisfied. Accordingly, we consider that the overall error function $E$ to be minimised in the $L_{2}$ sense can be expressed as the following weighted sum of four terms:

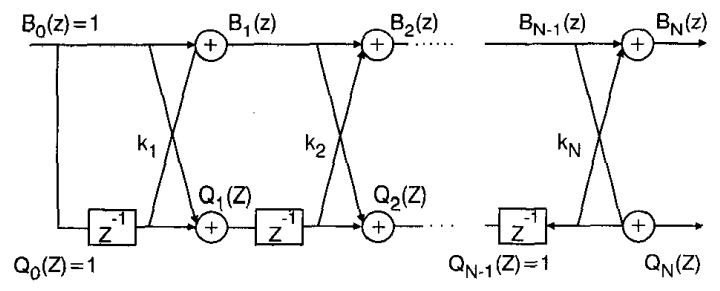

Fig. 3 Lattice structure for Nth-order FIR lattice filter

$$
\begin{aligned}
E= & \int_{\omega=0}^{\pi}\left|e^{-j k_{d}(\omega}-\hat{T}\left(e^{j \omega}\right)\right|^{2} d \omega \\
& +\alpha \int_{\omega=0}^{\omega=\omega_{p}}\left|H_{1}\left(e^{j \omega}\right)\right|^{2} d \omega+\beta \int_{\omega=\omega_{s}}^{\pi}\left|H_{0}\left(e^{j \omega}\right)\right|^{2} d \omega \\
& +\gamma \int_{\omega=\omega_{p}}^{\omega=\omega_{s}} \mid \frac{1}{L L_{0}} H_{0}\left(e^{j \omega}\right) H_{0}\left(e^{j\left(\omega-\omega_{p}-\omega_{s}\right)}\right) \\
& -\left.\frac{1}{L L_{1}} H_{1}\left(e^{j\left(\omega-\omega_{p}-\omega_{s}\right)}\right) H_{1}\left(e^{j \omega}\right)\right|^{2} d \omega
\end{aligned}
$$

where $\alpha, \beta$, and $\gamma$ represent the relative weights between the four error terms. We note from eqn. 11 that the overall error function $E$ is a function of fourth degree in the tap and reflection coefficients. Therefore, minimising $E$ directly leads to a very highly nonlinear programming problem in addition to the stability problem for $H_{0}(z)$ and $H_{1}(z)$.

\subsection{Proposed design technique}

Let $\left\{\omega_{1}=0, \omega_{2}, \ldots, \omega_{I}=\omega_{p}, \omega_{I+1}, \ldots, \omega_{I+J}, \omega_{I+J+1}\right.$ $\left.=\omega_{s}, \ldots, \omega_{I+J+K}=\pi\right\}$ be a grid of equidistant frequencies, distributed in the range of $\omega=0$ to $\omega=\pi$, for evaluating the magnitude response of the NDF bank and the related error terms defined as above. Moreover, assume that the set $S_{1}$ contains the grid points $\left\{\omega_{1}=0, \omega_{2}, \ldots\right.$, $\left.\omega_{I}=\omega_{p}\right\}, S_{2}$ contains the grid points $\left\{\omega_{I+1}, \omega_{I+2}\right.$, $\left.\ldots, \omega_{I+J}\right\}$, and $S_{3}$ contains the grid points $\left\{\omega_{I+J+1}\right.$ $\left.=\omega_{s}, \omega_{I+J+2}, \ldots, \omega_{I+J+K}=\pi\right\}$. We then rewrite eqn. 11 as the following approximation:

$$
\begin{aligned}
\hat{E}= & \sum_{\omega_{i} \in S_{1} \cup S_{2} \cup S_{3}}\left|e^{-j k_{d} \omega_{i}}-\hat{T}\left(e^{j \omega_{i}}\right)\right|^{2} \\
& +\alpha \sum_{\omega_{i} \in S_{1}}\left|H_{1}\left(e^{j \omega_{i}}\right)\right|^{2}+\beta \sum_{\omega_{i} \in S_{3}}\left|H_{0}\left(e^{j \omega \omega_{i}}\right)\right|^{2} \\
& +\gamma \sum_{\omega_{i} \in \omega_{p} \cup S_{2} \cup \omega_{s}} \mid \frac{1}{L L_{0}} H_{0}\left(e^{j \omega}\right) H_{0}\left(e^{j\left(\omega-\omega_{p}-\omega_{s}\right)}\right) \\
& -\left.\frac{1}{L L_{1}} H_{1}\left(e^{j\left(\omega-\omega_{p}-\omega_{s}\right)}\right) H_{1}\left(e^{j \omega}\right)\right|^{2}
\end{aligned}
$$

3.2.1 Determination of initial guesses for $H_{0}(z)$ and $H_{1}(z)$ : Our design experience shows that using better initial guesses for $H_{0}\left(e^{j \omega}\right)$ and $H_{1}\left(e^{j \omega}\right)$ usually provides better design results. To initiate the design process, we propose a procedure for determining appropriate initial guesses $H_{0}^{0}(z)$ and $H_{1}^{0}(z)$ for $H_{0}(z)$ and $H_{1}(z)$, respectively. According to the principle of a two-channel NDF bank, we define two desired frequency responses $D_{0}\left(e^{j \omega}\right)$ and $D_{1}\left(e^{j \omega}\right)$ as

$$
\begin{gathered}
D_{0}\left(e^{j \omega}\right)=\left\{\begin{array}{c}
\sqrt{L L_{0}} e^{-j \frac{k_{d}}{2}(\omega)}, \text { for } \omega \in\left[0, \omega_{p}\right] \\
\sqrt{\frac{L L_{0}}{2}} e^{-j \frac{k_{d}}{2} \omega}, \text { for } \omega=\frac{\omega_{p}+\omega_{s}}{2} \\
0, \text { for } \omega \in\left[\omega_{s}, \pi\right]
\end{array}\right. \\
D_{1}\left(e^{j \omega}\right)=\left\{\begin{array}{c}
0, \text { for } \omega \in\left[0, \omega_{p}\right] \\
j \sqrt{\frac{L L_{1}}{2}} e^{-j \frac{k_{d} \omega}{2},}, \text { for } \omega=\frac{\omega_{p}+\omega_{s}}{2} \\
j \sqrt{L L_{1}} e^{-j \frac{k_{d}}{2}(\omega)}, \text { for } \omega \in\left[\omega_{s}, \pi\right]
\end{array}\right.
\end{gathered}
$$

Two FIR filters $G_{0}(z)$ and $G_{1}(z)$ with orders $2 N_{0}$ and $2 N_{1}$ are designed to optimally approximate $D_{0}\left(e^{j \omega}\right)$ and $D_{1}\left(e^{j \omega}\right)$,

IEE Proc.-Vis. Image Signal Process., Vol. 147, No. 6, December 2000 
Table 1: Significant design results for example 1

\begin{tabular}{lll}
\hline & IIR NDF bank & FIR NDF bank \\
\hline Filter order & $10 / 10,11 / 11$ & 32,33 \\
No. of coefficients & 44 & 67 \\
PRE in $\left|T\left(e^{j \omega}\right)\right|(\mathrm{dB})$ & 0.0148 & 0.0238 \\
Max. variation of group & 0.0583 & 0.0746 \\
delay of $T\left(e^{j \omega}\right)$ & & \\
NPSR $(\mathrm{dB})$ of $H_{0}\left(e^{j \omega}\right)$ & 32.21 & 32.10 \\
NPSR $(\mathrm{dB})$ of $H_{1}\left(e^{j \omega}\right)$ & 32.07 & 31.95 \\
Max. variation of passband & 0.0158 & 0.0160 \\
group delay of $H_{0}\left(e^{j \omega}\right)$ & & 0.0163 \\
Max. variation of passband & 0.0230 & $3.85 \times 10^{-2}$ \\
group delay of $H_{1}\left(e^{j \omega}\right)$ & & $5.12 \times 10^{-2}$ \\
SEE of $H_{0}\left(e^{j \omega}\right)$ & $3.33 \times 10^{-2}$ & $2.76 \times 10^{-3}$ \\
SEE of $H_{1}\left(e^{j \omega}\right)$ & $5.68 \times 10^{-2}$ & 9 \\
Max. variation of filter & $2.27 \times 10^{-3}$ & \\
bank response & & 37 \\
No. of iterations & & \\
\hline
\end{tabular}

respectively, using conventional least-squares error criteria. Let the resulting filter coefficients be given by $\left\{h_{00}, h_{01}, \ldots\right.$, $\left.h_{0\left(2 N_{0}\right)}\right\}$ and $\left\{h_{10}, h_{11}, \ldots, h_{1\left(2 N_{1}\right)}\right\}$, respectively.

Through the use of the model reduction algorithm presented previously [13], we find two IIR filters where the numerator and denominator are of order $N_{0}$ (corresponding to $G_{0}(z)$ ) and of order $N_{1}$ (corresponding to $G_{1}(z)$ ), respectively. Assume that the IIR filters have denominators $C_{0}(z)$ and $C_{1}(z)$ with coefficients $\left\{c_{00}=1\right.$, $\left.c_{01}, \ldots, c_{0 N_{0}}\right\}$ and $\left\{c_{10}=1, c_{11}, \ldots, c_{1 N_{1}}\right\}$, respectively. The initial lattice systems $B_{N_{0}}^{0}$ and $B_{N_{1}}^{0}$ with reflection coefficients $\left\{k_{01}^{0}, k_{02}^{0}, \ldots, k_{0 N_{0}}^{0}\right\}$ and $\left\{k_{11}^{0}, k_{12}^{0}, \ldots, k_{1 N_{1}}^{0}\right\}$ corresponding to $C_{0}(z)$ and $C_{1}(z)$, respectively, can then be found since there exists a one-to-one correspondence between $\left\{c_{i 0}, c_{i 1}, \ldots, c_{i N_{i}}\right\}$ and $\left\{k_{i 1}^{0}, k_{i 2}^{0}, \ldots, k_{i N_{i}}^{0}\right\}[14]$ for $i=0,1$.

The best $L_{2}$ solution for the corresponding initial numerator $A_{i}^{0}(z), i=0,1$, can be obtained by solving the following optimisation problem:

$$
\begin{aligned}
& \text { minimise }\left|D_{i}\left(e^{j \omega}\right)-\frac{A_{i}^{0}\left(e^{j \omega}\right)}{B_{N_{i}}^{0}\left(e^{j \omega}\right)}\right|^{2}, \\
& \text { for all } \omega \in\left[0, \omega_{p}\right] \cup \frac{\omega_{p}+\omega_{s}}{2} \cup\left[\omega_{s}, \pi\right]
\end{aligned}
$$

For evaluating the related error functions given by eqn. 15 , we again take a set of discrete frequency points linearly distributed over $S=\left[0, \omega_{p}\right] \cup\left(\omega_{p}+\omega_{s}\right) / 2 \cup\left[\omega_{s}, \pi\right]$. Let $S_{d}=S_{1} \cup\left(\omega_{p}+\omega_{s}\right) / 2 \cup S_{3}=\left\{\omega_{1}=0, \omega_{2}, \ldots, \omega_{I}=\omega_{p}\right.$, $\left.\omega_{I+1}=\left(\omega_{p}+\omega_{s}\right) / 2, \quad \omega_{I+2}=\omega_{s}, \ldots, \omega_{I+K+1}=\pi\right\}$ be the dense grid of frequency points, and $\boldsymbol{U}_{i}$ be a complex $(I+$ $K+1) \times\left(M_{i}+1\right)$ matrix with its $(m, n)$ th element given by

$$
\begin{array}{r}
U_{i}(m, n)=\frac{e^{-j(n-1) \omega_{m}}}{B_{N_{i}}^{0}\left(e^{\left.j \omega_{m}\right)}\right.}, 1 \leq m \leq I+K+1, \\
1 \leq n \leq M_{i}+1
\end{array}
$$

$\boldsymbol{d}_{i}$ is a complex $(I+K+1) \times 1$ vector, with its $m$ th element given by

$$
d_{i}(m)=D_{i}\left(e^{j \omega_{m}}\right), \quad 1 \leq m \leq I+K+1
$$

The initial coefficient vector $\boldsymbol{a}_{i}^{0}=\left[a_{i 0}^{0}, a_{i 1}^{0}, \ldots, a_{i M_{i}}^{0}\right]^{T}$ of $A_{i}^{0}(z)$, optimal in the $L_{2}$ sense for eqn. 15 , can be found by minimising $\left|\boldsymbol{U}_{i} \boldsymbol{a}_{i}^{0}-\boldsymbol{d}_{i}\right|^{2}=\left(\boldsymbol{U}_{i} \boldsymbol{a}_{i}^{0}-\boldsymbol{d}_{i}\right)^{H}\left(\boldsymbol{U}_{i} \boldsymbol{a}_{i}^{0}-\boldsymbol{d}_{i}\right)$, where ${ }^{H}$ denotes the complex conjugate transpose. Clearly, this leads to the optimal solution given by $\boldsymbol{a}_{i}^{0}=\left\{\operatorname{Re}\left(\boldsymbol{U}_{i}^{H} \boldsymbol{U}_{i}\right)\right\}^{-1}\left[\operatorname{Re}\left(\boldsymbol{U}_{i}^{\boldsymbol{H}} \boldsymbol{d}_{i}\right)\right]$, for $i=0,1$, where $\operatorname{Re}(\boldsymbol{X})$ represents the real part of the matrix $X$. After finding the appropriate initial guesses $H_{i}^{0}(z)=A_{i}^{0}(z) / B_{N_{i}}^{0}$, we present an iterative procedure step by step for computing $A_{i}(z)$ and $B_{N_{i}}(z)$, for $i=0,1$, during the design process.

\subsubsection{Iterative procedure:}

Step 1: At the lth iteration, let the analysis filters be

$$
H_{0}^{l}\left(e^{j \omega}\right)=\frac{A_{0}^{l}\left(e^{j \omega}\right)}{B_{N_{0}}^{l}\left(e^{j \omega}\right)} \quad \text { and } \quad H_{1}^{l}\left(e^{j \omega}\right)=\frac{A_{1}^{l}\left(e^{j \omega}\right)}{B_{N_{1}}^{l}\left(e^{j \omega}\right)}
$$

Utilise a linearisation scheme to rewrite $\hat{T}\left(e^{j \omega_{i}}\right)$ and $\hat{V}\left(e^{j \omega_{i}}\right)$ as follows [15]:

\begin{tabular}{|c|c|c|c|c|}
\hline$i$ & $a_{0 i}$ & $k_{0 i}$ & $a_{1 i}$ & $k_{1 i}$ \\
\hline 0 & $8.6657905 \times 10^{-3}$ & & $-6.6212287 \times 10^{-3}$ & \\
\hline 1 & $-4.0416626 \times 10^{-2}$ & $-5.2321871 \times 10^{-1}$ & $-2.6735861 \times 10^{-2}$ & $-1.0973161 \times 10^{-1}$ \\
\hline 2 & $7.2162064 \times 10^{-2}$ & $7.7911067 \times 10^{-1}$ & $-4.8663890 \times 10^{-2}$ & $6.5122570 \times 10^{-1}$ \\
\hline 3 & $-5.6558510 \times 10^{-2}$ & $-7.2660247 \times 10^{-1}$ & $-3.8462788 \times 10^{-2}$ & $4.7095441 \times 10^{-1}$ \\
\hline 4 & $3.0787734 \times 10^{-2}$ & $6.7708888 \times 10^{-1}$ & $2.2971930 \times 10^{-2}$ & $4.0467721 \times 10^{-1}$ \\
\hline 5 & $-5.9047004 \times 10^{-2}$ & $-6.3779579 \times 10^{-1}$ & $8.0657319 \times 10^{-2}$ & $3.5518737 \times 10^{-1}$ \\
\hline 6 & $2.9256295 \times 10^{-2}$ & $5.8390526 \times 10^{-1}$ & $1.3217710 \times 10^{-2}$ & $3.1592069 \times 10^{-1}$ \\
\hline 7 & $8.6821333 \times 10^{-2}$ & $-4.5798891 \times 10^{-1}$ & $-2.2281802 \times 10^{-1}$ & $2.7136095 \times 10^{-1}$ \\
\hline 8 & $1.6613748 \times 10^{-1}$ & $2.4768711 \times 10^{-1}$ & $-3.2913158 \times 10^{-1}$ & $2.0592667 \times 10^{-1}$ \\
\hline 9 & $1.1964706 \times 10^{-1}$ & $-6.9787484 \times 10^{-2}$ & $1.3735270 \times 10^{-0}$ & $1.2434145 \times 10^{-1}$ \\
\hline 10 & $8.9118083 \times 10^{-2}$ & $6.0013386 \times 10^{-3}$ & $-1.4431611 \times 10^{-0}$ & $5.2666408 \times 10^{-2}$ \\
\hline 11 & & & $6.2466860 \times 10^{-1}$ & $1.2187899 \times 10^{-2}$ \\
\hline
\end{tabular}

$$
\begin{aligned}
\tilde{T}\left(e^{j \omega_{i}}\right)= & \frac{1}{L L_{0}} H_{0}^{l}\left(e^{j \omega_{i}}\right) \frac{\tilde{A}_{0}\left(e^{j \omega_{i}}\right)}{B_{N_{0}}^{l}\left(e^{j \omega_{i}}\right)} \\
& -\frac{1}{L L_{1}} H_{1}^{l}\left(e^{j \omega_{i}}\right) \frac{\tilde{A}_{1}\left(e^{j \omega_{i}}\right)}{B_{N_{1}}^{l}\left(e^{j \omega_{i}}\right)}
\end{aligned}
$$

and

$$
\begin{aligned}
\tilde{V}\left(e^{j \omega_{i}}\right)= & \frac{1}{L L_{0}} H_{0}^{l}\left(e^{j\left(\omega_{i}-\omega_{p}-\omega_{s}\right)}\right) \frac{\tilde{A}_{0}\left(e^{j \omega_{i}}\right)}{B_{N_{0}}^{l}\left(e^{j \omega_{i}}\right)} \\
& -\frac{1}{L L_{1}} H_{1}^{l}\left(e^{j\left(\omega_{i}-\omega_{p}-\omega_{s}\right)}\right) \frac{\tilde{A}_{1}\left(e^{j \omega_{i}}\right)}{B_{N_{1}}^{l}\left(e^{j \omega_{i}}\right)}
\end{aligned}
$$

Table 2: Tap and reflection coefficients for example 1 
respectively, where the numerators $\tilde{A}_{i}\left(e^{j \omega_{i}}\right)$ have coefficient vectors $\tilde{\boldsymbol{a}}_{i}=\left[\tilde{a}_{i 0}, \tilde{a}_{i 1}, \ldots, \tilde{a}_{i M}\right]^{T}$, for $i=0,1$. We then find the optimal numerators $\tilde{A}_{i}\left(e^{j \omega_{i}}\right)$. First, several matrices are defined as follows: $\boldsymbol{W}_{0}$ is an $(I+J+K) \times\left(M_{0}+1\right)$ matrix, with the $(m, n)$ th entry given by

$$
\begin{aligned}
W_{0}(m, n)= & \frac{1}{L L_{0}} H_{0}^{l}\left(e^{j \omega_{m}}\right) \frac{e^{-j(n-1) \omega_{m}}}{B_{N_{0}}^{l}\left(e^{j \omega_{m}}\right)}, \\
& \omega_{m} \in S_{1} \cup S_{2} \cup S_{3}, n=1,2, \ldots, M_{0}+1
\end{aligned}
$$

$\boldsymbol{W}_{1}$ is an $(I+J+K) \times\left(M_{1}+1\right)$ matrix, with the $(m, n)$ th entry given by

$$
\begin{aligned}
W_{1}(m, n)= & -\frac{1}{L L_{1}} H_{1}^{l}\left(e^{j \omega_{m}}\right) \frac{e^{-j(n-1) \omega_{m}}}{B_{N_{1}}^{l}\left(e^{j \omega_{m}}\right)}, \\
& \omega_{m} \in S_{1} \cup S_{2} \cup S_{3}, n=1,2, \ldots, M_{1}+1
\end{aligned}
$$

$\boldsymbol{Y}_{0}$ is an $K \times\left(M_{0}+1\right)$ matrix, with the $(m, n)$ th entry given by

$$
\begin{aligned}
Y_{0}(m, n)= & \frac{e^{-j(n-1) \omega_{m+l+J}}}{B_{N_{0}}^{l}\left(e^{\left.j \omega_{m+I+J}\right)}\right.}, \\
& \omega_{m+I+J} \in S_{3}, n=1,2, \ldots, M_{0}+1
\end{aligned}
$$

$\boldsymbol{Y}_{1}$ is an $I \times\left(M_{1}+1\right)$ matrix, with the $(m, n)$ th entry given by

$$
Y_{1}(m, n)=\frac{e^{-j(n-1) \omega_{m}}}{B_{N_{1}}^{l}\left(e^{\left.j \omega_{m}\right)}\right.}, \omega_{m} \in S_{1}, n=1,2, \ldots, M_{1}+1
$$

$\boldsymbol{Z}_{0}$ is an $(J+2) \times\left(M_{0}+1\right)$ matrix, with the $(m, n)$ th entry given by

$$
\begin{aligned}
Z_{0}(m, n)= & \frac{H_{0}^{l}\left(e^{j\left(\omega_{n}-\omega_{p}-\omega_{s}\right)}\right)}{L L_{0}} \frac{e^{-j(n-1) \omega_{m+l-1}}}{B_{N_{0}}^{l}\left(e^{j \omega_{m+l-1}}\right)}, \\
& \omega_{m} \in \omega_{p} \cup S_{2} \cup \omega_{s}, n=1,2, \ldots, M_{0}+1
\end{aligned}
$$

$Z_{1}$ is an $(J+2) \times\left(M_{1}+1\right)$ matrix, with the $(m, n)$ th entry given by

$$
\begin{aligned}
& Z_{1}(m, n)=-\frac{H_{1}^{l}\left(e^{j\left(\omega_{m+l-1}-\omega_{p}-\omega_{s}\right)}\right)}{L L_{1}} \frac{e^{-j(n-1) \omega_{m+l-1}}}{B_{N_{1}}^{l}\left(e^{j \omega_{m+l-1}}\right)}, \\
& \omega_{m} \in \omega_{p} \cup S_{2} \cup \omega_{s}, n=1,2, \ldots, M_{1}+1
\end{aligned}
$$

Moreover, $\boldsymbol{W}=\left[\begin{array}{ll}\boldsymbol{W}_{0}, & \boldsymbol{W}_{1}\end{array}\right], \boldsymbol{Y}_{00}=\left[\begin{array}{ll}\boldsymbol{Y}_{0}, & \mathbf{0}_{K \times\left(M_{1}+1\right)}\end{array}\right], \boldsymbol{Y}_{11}=$ $\left[\mathbf{0}_{I \times\left(M_{0}+1\right)}, \boldsymbol{Y}_{1}\right]$, where $\mathbf{0}_{M \times N}$ denotes a zero matrix with size shown by its subscript $M \times N, \boldsymbol{Z}=\left[\boldsymbol{Z}_{0}, \boldsymbol{Z}_{1}\right]$, and $z_{d}$ is an $(I+J+K) \times 1$ complex vector, with the $m$ th entry given by

$$
z_{d}(m)=e^{-j k_{d} \omega_{m}}, \omega_{m} \in S_{1} \cup S_{2} \cup S_{3}
$$

As a result, the required coefficient vectors $\tilde{\boldsymbol{a}}_{i}$ can be found from eqn. 12 by minimising the following error function:

$$
\begin{aligned}
\tilde{E}= & \left(\boldsymbol{W} \tilde{\boldsymbol{a}}-\boldsymbol{z}_{d}\right)^{H}\left(\boldsymbol{W} \tilde{\boldsymbol{a}}-\boldsymbol{z}_{d}\right)+\alpha\left(\boldsymbol{Y}_{11} \tilde{\boldsymbol{a}}\right)^{H}\left(\boldsymbol{Y}_{11} \tilde{\boldsymbol{a}}\right) \\
& +\beta\left(\boldsymbol{Y}_{00} \tilde{\boldsymbol{a}}\right)^{H}\left(\boldsymbol{Y}_{00} \tilde{\boldsymbol{a}}\right)+\gamma(\boldsymbol{Z} \tilde{\boldsymbol{a}})^{H}(\boldsymbol{Z} \tilde{\boldsymbol{a}})
\end{aligned}
$$
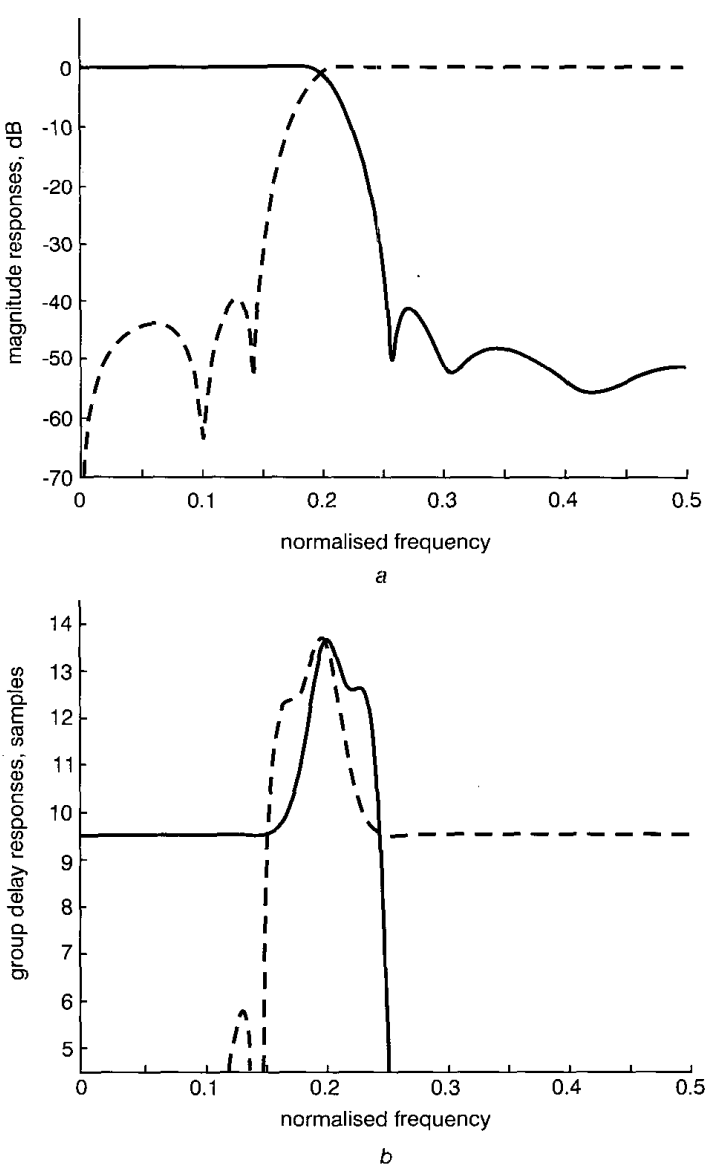

Fig. 4 Magnitude and group delay responses of analysis filters for example 1

$-H_{0}\left(e^{j \omega)}\right) / \sqrt{ }\left(L L_{0}\right)$
$--\quad H_{1}\left(e^{j \omega)}\right) / \sqrt{ }\left(L L_{1}\right)$
a Magnitude response

$b$ Group delay response

where the vector $\tilde{\boldsymbol{a}}=\left[\tilde{\boldsymbol{a}}_{0}^{T}, \tilde{\boldsymbol{a}}_{1}^{T}\right]^{T}$. Clearly, the optimal solution for eqn. 26 is given by

$$
\begin{aligned}
\tilde{\boldsymbol{a}}= & \left\{\operatorname{Re}\left[\boldsymbol{W}^{H} \boldsymbol{W}+\alpha \boldsymbol{Y}_{11}^{H} \boldsymbol{Y}_{11}+\beta \boldsymbol{Y}_{00}^{H} \boldsymbol{Y}_{00}+\gamma \boldsymbol{Z}^{H} \boldsymbol{Z}\right]\right\}^{-1} \\
& \times\left\{\operatorname{Re}\left[\boldsymbol{W}^{H} \boldsymbol{z}_{d}\right]\right\}
\end{aligned}
$$

since eqn. 26 is a quadratic function of the coefficients $\tilde{a}_{i 0}$, $\tilde{a}_{i 1}, \ldots, \tilde{a}_{i M_{i}}$, for $i=0,1$. After obtaining the coefficient vectors $\tilde{\boldsymbol{a}}_{i}$, we update the numerator coefficients of $H_{i}^{l}(z)$ as follows:

$$
\boldsymbol{a}_{i}^{l+1}=(1-\tau) \boldsymbol{a}_{i}^{l}+\tau \tilde{\boldsymbol{a}}_{i}
$$

for $i=0$, 1, where $\tau(0<\tau<1)$ is a smoothing parameter with the best value chosen experimentally.

Step 2: Compute the gradient matrices $\boldsymbol{H}_{t}=\left[\nabla \hat{T}^{l}\left(e^{j \omega_{i}}\right)\right]$ for $\omega_{i} \in S_{1} \cup S_{2} \cup S_{3} . \boldsymbol{H}_{0}=\left[\nabla H_{0}^{l}\left(e^{j \omega_{i}}\right)\right]$ for $\omega_{i} \in S_{3}, \boldsymbol{H}_{1}=$ $\left[\nabla H_{1}^{l}\left(e^{j \omega_{i}}\right)\right]$ for $\omega_{i} \in S_{1}$, and $\boldsymbol{H}_{a}=\left[\nabla \hat{V}^{l}\left(e^{j \omega_{t}}\right)\right]$ for $\omega_{i} \in \omega_{p}$ $\cup S_{2} \cup \omega_{s}$, where $\nabla$ represents the gradient operator $\left[\partial / \partial k_{01}, \partial / \partial k_{02}, \ldots, \quad \partial / \partial k_{0 N_{0}}, \partial / \partial a_{00}, \partial / \partial a_{01}, \ldots, \partial / \partial a_{0 M_{0}}\right.$, $\left.\partial / \partial k_{11}, \partial / \partial k_{12}, \ldots, \partial / \partial k_{1 N_{1}}, \partial / \partial a_{10}, \partial / \partial a_{11}, \ldots, \partial / \partial a_{1 M_{\mathrm{l}}}\right]$ Step 3: Use a linearisation scheme to approximate the frequency response error $\hat{E}^{\prime}$ for eqn. 12 due to a perturbation in the coefficient vector in the linear subspace spanned

IEE Proc.-Vis. Image Signal Process., Vol. 147, No. 6, December 2000 


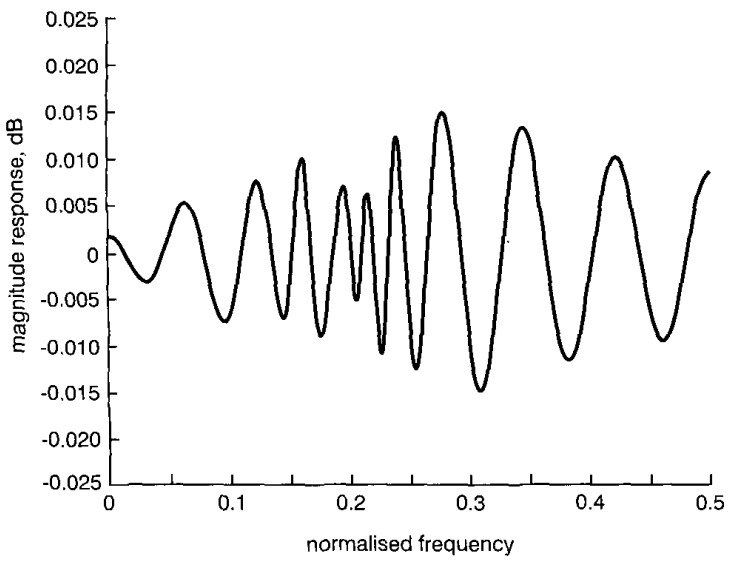

a

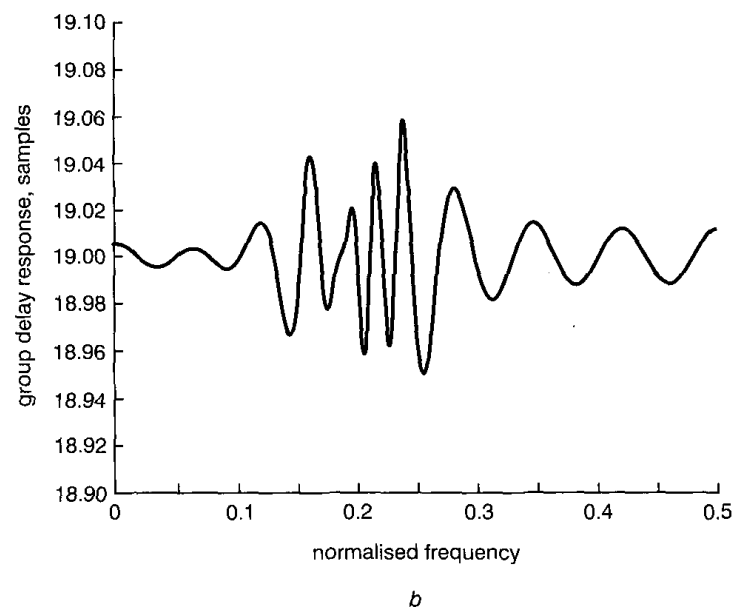

Fig. 5 Magnitude and group delay responses of $\hat{T}\left(e^{j \omega}\right)$ for example 1 a Magnitude response $b$ Group delay response

by the gradient matrices $\boldsymbol{H}_{t}, \boldsymbol{H}_{0}, \boldsymbol{H}_{1}$, and $\boldsymbol{H}_{\boldsymbol{a}}$. This means that the approximation error given by

$$
\begin{aligned}
\hat{E}_{a}^{l}= & \sum_{\omega_{i} \in S_{1} \cup S_{2} \cup S_{3}}\left|e^{-j k_{d} \omega_{i}}-\hat{T}^{l}\left(e^{j \omega_{i}}\right)-\nabla \hat{T}^{l}\left(e^{j \omega_{i}}\right) \boldsymbol{v}\right|^{2} \\
& +\alpha \sum_{\omega_{i} \in S_{1}}\left|H_{1}^{l}\left(e^{j \omega_{i}}\right)+\nabla H_{1}^{l}\left(e^{j \omega_{i}}\right) \boldsymbol{v}\right|^{2} \\
& +\beta \sum_{\omega_{i} \in S_{3}}\left|H_{0}^{l}\left(e^{j \omega_{i}}\right)+\nabla H_{0}^{l}\left(e^{j \omega_{i}}\right) v\right|^{2} \\
& +\gamma \sum_{\omega_{i} \in \omega_{p} \cup S_{2} \cup \omega_{s}}\left|\hat{V}^{l}\left(e^{j \omega_{i}}\right)+\nabla \hat{V}^{l}\left(e^{j \omega_{i}}\right) v\right|^{2}
\end{aligned}
$$

is computed, where the vector $\boldsymbol{v}=\left[\Delta \boldsymbol{k}_{0}, \Delta a_{0}, \Delta \boldsymbol{k}_{1}, \Delta \boldsymbol{a}_{1}\right]=$ $\left[\Delta k_{01}, \Delta k_{02}, \ldots, \Delta k_{0 N_{0}}, \Delta a_{00}, \Delta a_{01}, \ldots, \Delta a_{0 M_{0}}, \Delta k_{11}\right.$, $\left.\Delta k_{12}, \ldots, \Delta k_{1 N_{1}}, \Delta a_{10}, \Delta a_{11}, \ldots, \Delta a_{1 M_{1}}\right]^{T}$ contains the increments of the independent coefficients to be found.

We solve the minimisation problem of eqn. 29 to obtain the increment coefficient vector $\boldsymbol{v}$. Let $\boldsymbol{r}_{1}$ be an $(I+$ $J+K) \times 1$ complex vector, with the $i$ th entry given by $r_{1}(i)=e^{-j k_{d} \omega_{i}}-\hat{T}^{l}\left(e^{j \omega_{i}}\right)$, for $\omega_{i} \in S_{1} \cup S_{2} \cup S_{3} ; \boldsymbol{r}_{2}$ be an $I \times 1$ complex vector, with the $i$ th entry given by $r_{2}(i)=-H_{1}^{l}\left(e^{j \omega_{i}}\right)$, for $\omega_{i} \in S_{1} ; r_{3}$ be a $K \times 1$ complex vector, with the $i$ th entry given by $r_{3}(i)=-H_{0}^{l}\left(e^{j \omega_{i+l+J}}\right)$, for $\omega_{i+I+J} \in S_{3}$; and $\boldsymbol{r}_{4}$ be a $(J+2) \times 1$ complex vector,

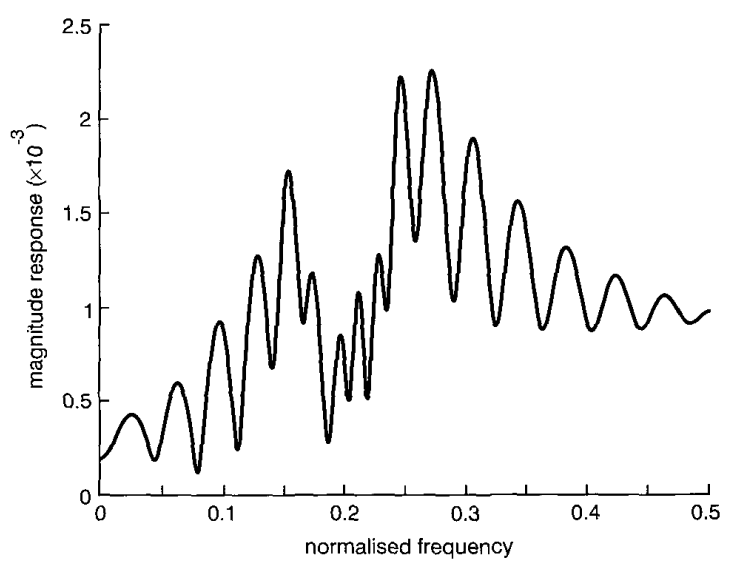

Fig. 6 Magnitude response of variation of filter bank response for example 1

with the $i$ th entry given by $r_{4}(i)=-\hat{V}^{l}\left(e^{j \omega_{i+1-1}}\right)$, for $\omega_{i+l-1} \in \omega_{p} \cup S_{2} \cup \omega_{s}$. The optimal solution $v$ for eqn. 29 can then be expressed as

$$
\begin{aligned}
\boldsymbol{v}= & \left\{\operatorname{Re}\left[\boldsymbol{H}_{t}^{H} \boldsymbol{H}_{t}+\alpha \boldsymbol{H}_{1}^{H} \boldsymbol{H}_{1}+\beta \boldsymbol{H}_{0}^{H} \boldsymbol{H}_{0}+\gamma \boldsymbol{H}_{a}^{H} \boldsymbol{H}_{a}\right]\right\}^{-1} \\
& \times \operatorname{Re}\left[\boldsymbol{H}_{t}^{H} \boldsymbol{r}_{1}+\alpha \boldsymbol{H}_{1}^{H} \boldsymbol{r}_{2}+\beta \boldsymbol{H}_{0}^{H} \boldsymbol{r}_{3}+\gamma \boldsymbol{H}_{a}^{H} \boldsymbol{r}_{4}\right]
\end{aligned}
$$

Step 4: Perform a line search by using the Nelder-Meade simplex algorithm [16] to find the best step size $t$ with $0<t<1$, to update the numerators and denominators of $H_{0}(z)$ and $H_{1}(z)$ such that the following error function reaches its minimum:

$$
\begin{aligned}
\hat{E}^{l+1}= & \sum_{\omega_{i} \in S_{1} \cup S_{2} \cup S_{3}}\left|e^{-j k_{d} \omega_{i}}-\hat{T}^{l+1}\left(e^{j \omega_{i}}\right)\right|^{2} \\
& +\alpha \sum_{\omega_{i} \in S_{1}}\left|H_{1}^{l+1}\left(e^{j \omega_{i}}\right)\right|^{2} \\
& +\beta \sum_{\omega_{i} \in S_{3}}\left|H_{0}^{l+1}\left(e^{j \omega_{i}}\right)\right|^{2} \\
& +\gamma \sum_{\omega_{i} \in \omega_{p} \cup S_{2} \cup \omega_{s}}\left|\hat{V}^{l+1}\left(e^{j \omega_{i}}\right)\right|^{2}
\end{aligned}
$$

subject to the constraints of $\max \left|k_{i j}^{l}+t \Delta k_{i j}\right|<k_{\max }$, for $i=0, j=1,2, \ldots, N_{0}$, and $i=1, j=1,2, \ldots, N_{1}$; where $H_{0}^{l+1}\left(e^{j \omega}\right)$ and $H_{1}^{l+1}\left(e^{j \omega}\right)$ have tap and reflection coefficient vectors given by $\boldsymbol{a}_{0}^{l+1}=\left[a_{00}^{l}+t \Delta a_{00}, a_{01}^{l}+t \Delta\right.$ $\left.a_{01}, \ldots, a_{0 M_{0}}^{l}+t \Delta a_{0 M_{0}}\right]^{T} ; \boldsymbol{k}_{0}^{l+1}=\left[k_{01}^{l}+t \Delta k_{01}, k_{02}^{l}+t \Delta k_{02}\right.$, $\left.\ldots, k_{0 N_{0}}^{l}+t \Delta k_{0 N_{0}}\right]^{T} ; \boldsymbol{a}_{1}^{l+1}=\left[a_{10}^{l}+t \Delta a_{10}, a_{11}^{l}+t \Delta a_{11}, \ldots\right.$, $\left.a_{1 M_{1}}^{l}+t \Delta a_{1 M_{1}}\right]^{T}$; and $\boldsymbol{k}_{1}^{l+1}=\left[k_{11}^{l}+t \Delta k_{11}, k_{12}^{l}+t \Delta k_{12}, \ldots\right.$, $\left.k_{1 N_{1}}^{l}+t \Delta k_{1 N_{1}}\right]^{T}$, respectively. $\hat{T}^{l+1}\left(e^{j \omega}\right)$ and $\hat{V}^{l+1}\left(e^{j \omega_{i}}\right)$ are the corresponding filter bank response and aliasing distortion. Moreover, $k_{\max }$ is a preset maximal absolute value and must be less than 1 for the reflection coefficients, in order to ensure the stability of the designed IIR NDF bank.

Step 5: Compute the overall error function of eqn. 12 corresponding to $H_{0}^{l+1}\left(e^{j \omega}\right), H_{1}^{l+1}\left(e^{j \omega}\right) . \hat{T}_{1}^{l+1}\left(e^{j \omega}\right)$ and $\hat{V}_{1}^{l+1}\left(e^{j \omega}\right)$, which is in fact given by eqn. 31 .

Step 6: Compute the ratio $\left|\hat{E}^{l}-\hat{E}^{l+1}\right| / \hat{E}^{l}$. If this ratio is larger than a preset positive number $c$, then set $l=l+1$ and go to Step 1. Otherwise, we terminate the design process. Remarks: There are two situations where the gradient matrices $\boldsymbol{H}_{t}, \boldsymbol{H}_{0}, \boldsymbol{H}_{1}$, and $\boldsymbol{H}_{a}$ may degenerate.

Case 1: The columns of $\boldsymbol{H}_{t}, \boldsymbol{H}_{0}, \boldsymbol{H}_{1}$, and $\boldsymbol{H}_{a}$ are not linearly independent. The optimal solution for eqn. 29 is then not 
Table 3: Significant design results for example 2

\begin{tabular}{|c|c|c|}
\hline & IIR NDF bank & FIR NDF bank \\
\hline Filter order & $13 / 14,17 / 17$ & 49,49 \\
\hline No. of coefficients & 63 & 100 \\
\hline PRE in $j T\left(e^{j \omega}\right) j(d B)$ & 0.0176 & 0.0190 \\
\hline $\begin{array}{l}\text { Max. variation of group } \\
\text { delay of } T\left(e^{j \alpha}\right)\end{array}$ & 0.0703 & 0.0918 \\
\hline $\operatorname{NPSR}(\mathrm{dB})$ of $H_{0}\left(e^{j \omega}\right)$ & 40.88 & 40.53 \\
\hline NPSR $(\mathrm{dB})$ of $H_{1}\left(e^{j \omega}\right)$ & 40.66 & 40.42 \\
\hline $\begin{array}{l}\text { Max. variation of passband } \\
\text { group delay of } H_{0}\left(e^{j \omega}\right)\end{array}$ & 0.0091 & 0.0304 \\
\hline $\begin{array}{l}\text { Max. variation of passband } \\
\text { group delay of } H_{1}\left(e^{j \omega}\right)\end{array}$ & 0.0247 & 0.0250 \\
\hline SEE of $H_{0}\left(e^{j \omega}\right)$ & $4.05 \quad 10^{-3}$ & $2.99 \quad 10^{-3}$ \\
\hline SEE of $H_{1}\left(e^{j \omega}\right)$ & $7.4210^{-3}$ & $6.4510^{-3}$ \\
\hline $\begin{array}{l}\text { Max. variation of filter } \\
\text { bank response }\end{array}$ & $2.10 \quad 10^{-3}$ & $2.57 \quad 10^{-3}$ \\
\hline No. of iterations & 41 & 10 \\
\hline
\end{tabular}

unique. To find an appropriate optimal solution, we construct matrices $\boldsymbol{G}_{t}, \boldsymbol{G}_{0}, \boldsymbol{G}_{1}$, and $\boldsymbol{G}_{\alpha}$ by choosing the independent columns from $\boldsymbol{H}_{t}, \boldsymbol{H}_{0}, \boldsymbol{H}_{1}$, and $\boldsymbol{H}_{a}$ and a vector $\boldsymbol{u}$ by choosing the components of $\boldsymbol{v}$ corresponding to the independent columns. Then use $\boldsymbol{G}_{t}, \boldsymbol{G}_{0}, \boldsymbol{G}_{1}, \boldsymbol{G}_{a}$ and $\boldsymbol{u}$ to replace $\boldsymbol{H}_{t}, \boldsymbol{H}_{0}, \boldsymbol{H}_{1}, \boldsymbol{H}_{a}$, and $\boldsymbol{v}$ in eqn. 29. On the other hand, if only $\boldsymbol{H}_{t}$ (or $\boldsymbol{H}_{0}$ or $\boldsymbol{H}_{1}$ or $\boldsymbol{H}_{a}$ ) has columns not linearly independent, then we construct a matrix $\boldsymbol{G}_{t}$ (or $\boldsymbol{G}_{0}$ or $\boldsymbol{G}_{1}$ or $\boldsymbol{G}_{a}$ ) by replacing the elements of those columns that are not linearly independent with zero elements from $\boldsymbol{H}_{t}\left(\right.$ or $\boldsymbol{H}_{0}$ or $\boldsymbol{H}_{1}$ or $\boldsymbol{H}_{a}$ ). Then use $\boldsymbol{G}_{t}$ (or $\boldsymbol{G}_{0}$ or $\boldsymbol{G}_{1}$ or $\boldsymbol{G}_{a}$ ) to replace $\boldsymbol{H}_{t}$ (or $\boldsymbol{H}_{0}$ or $\boldsymbol{H}_{1}$ or $\boldsymbol{H}_{a}$ ) in eqn. 29 to obtain an appropriate optimal solution.

Case 2: At the 1 th iteration, the $i$ th reflection coefficient $k_{i}$ may have the absolute value equal to $k_{\max }$. To tackle this difficulty, we construct a vector $\boldsymbol{u}$ by eliminating $\Delta k_{i}$ of the

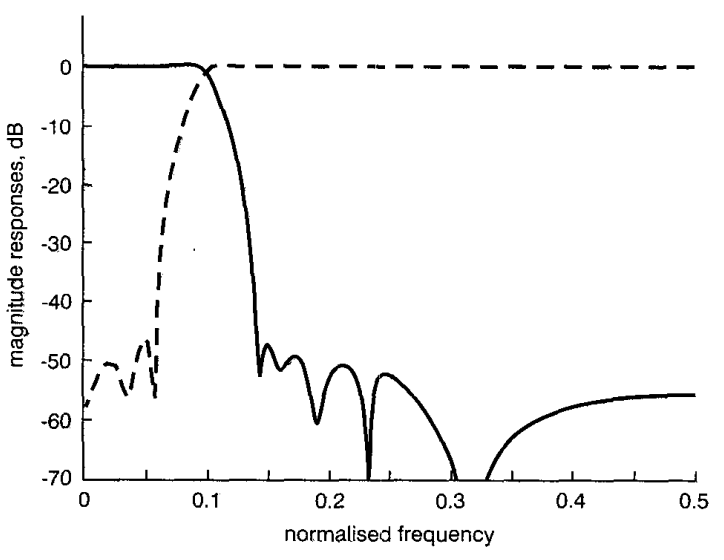

a

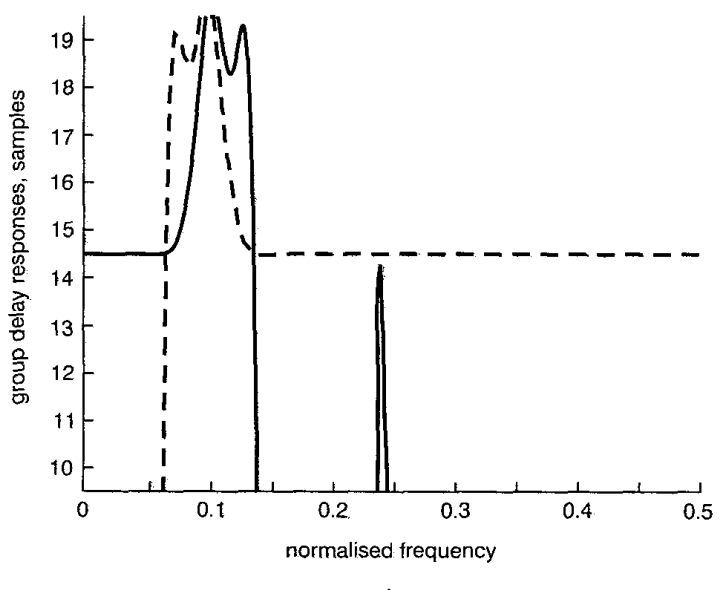

Fig. 7 Magnitude and group delay responses of analysis filters for example 2

$-\ldots H_{0}\left(e^{j \omega}\right) / \mathrm{p}\left(L L_{0}\right)$

- $\quad H_{1}\left(e^{j \omega}\right) /(L L)$

$a$ Magnitude response

Table 4: Tap and reflection coefficients for example 2

\begin{tabular}{|c|c|c|c|c|c|c|c|c|}
\hline$i$ & $a_{0 i}$ & & $k_{0 i}$ & & $a_{1 i}$ & & $k_{1 k}$ & \\
\hline 0 & -3.6798234 & $10^{-3}$ & & & 1.5980566 & $10^{-3}$ & & \\
\hline 1 & 2.6099284 & $10^{-2}$ & -8.2994053 & $10^{-1}$ & 3.5848626 & $10^{-3}$ & -8.1584134 & $10^{-1}$ \\
\hline 2 & -9.0796157 & $10^{-2}$ & 9.3802032 & $10^{-1}$ & 5.8518981 & $10^{-3}$ & 8.4751020 & $10^{-1}$ \\
\hline 3 & 2.1271854 & $10^{-1}$ & -8.7566468 & $.10^{-1}$ & 7.6135849 & $10^{-3}$ & 5.4108119 & $10^{-3}$ \\
\hline 4 & -3.8010473 & $10^{-1}$ & 8.5826413 & $10^{-1}$ & 7.1787741 & $10^{-3}$ & 1.1230581 & $10^{-1}$ \\
\hline 5 & 5.4977591 & $10^{-t}$ & -8.1937563 & $10^{-1}$ & 2.9788998 & $10^{-3}$ & 1.1278975 & $10^{-1}$ \\
\hline 6 & -6.6896321 & $10^{-1}$ & 7.4277861 & $10^{-1}$ & -5.9077057 & $10^{-3}$ & 1.0793339 & $10^{-1}$ \\
\hline 7 & 6.9851301 & $10^{-1}$ & -7.0493877 & $10^{-1}$ & -1.8788240 & $10^{-2}$ & 1.0367129 & $10^{-1}$ \\
\hline 8 & -6.2758842 & $10^{-1}$ & 7.0366242 & $10^{-1}$ & -3.2615090 & $10^{-2}$ & 9.8855147 & $10^{-2}$ \\
\hline 9 & 4.8330749 & $10^{-1}$ & -6.8684451 & $10^{-1}$ & -4.1421895 & $10^{-2}$ & 9.1518793 & $10^{-2}$ \\
\hline 10 & -3.0750743 & $10^{-1}$ & 6.8390959 & $10^{-1}$ & -3.5958718 & $10^{-2}$ & 8.0536860 & $10^{-2}$ \\
\hline 11 & 1.5743740 & $10^{-1}$ & -6.6734683 & $10^{-1}$ & -1.9035730 & $10^{-3}$ & 6.6165397 & $10^{-2}$ \\
\hline 12 & -5.6882050 & $10^{-2}$ & 5.5310626 & $10^{-1}$ & 8.9533637 & $10^{-2}$ & 4.9924064 & $10^{-2}$ \\
\hline 13 & 1.3209640 & $10^{-2}$ & -3.0505849 & $10^{-1}$ & 3.4236521 & $10^{-1}$ & 3.3990564 & $10^{-2}$ \\
\hline 14 & & & 7.3282216 & $10^{-2}$ & 1.8856250 & $10^{-0}$ & 2,0406182 & $10^{-2}$ \\
\hline 15 & & & & & -6.1255005 & $10^{-0}$ & 1.0412525 & $10^{-2}$ \\
\hline 16 & & & & & 5.6539967 & $10^{-0}$ & 4.2050121 & $10^{-3}$ \\
\hline 17 & & & & & -1.7337973 & $10^{-0}$ & 1.10958031 & $0^{-3}$ \\
\hline
\end{tabular}


vector $\boldsymbol{v}$ and four matrices $\boldsymbol{G}_{t}, \boldsymbol{G}_{0}, \boldsymbol{G}_{1}$, and $\boldsymbol{G}_{a}$ by eliminating the columns of $\boldsymbol{H}_{t}, \boldsymbol{H}_{0}, \boldsymbol{H}_{1}$, and $\boldsymbol{H}_{a}$, corresponding to $\Delta k_{i}$, respectively. Then, we use $\boldsymbol{G}_{t}, \boldsymbol{G}_{0}, \boldsymbol{G}_{1}, \boldsymbol{G}_{a}$, and $\boldsymbol{u}$ to replace $\boldsymbol{H}_{t}, \boldsymbol{H}_{0}, \boldsymbol{H}_{1}, \boldsymbol{H}_{a}$, and $\boldsymbol{v}$ in eqn. 29.

\section{Simulation examples}

In this Section, we present several simulation examples of designing two-channel IIR NDF banks for illustration. For all design examples, the spacing for two adjacent frequency points in $[0, \pi]$ is set to $\pi / 299$, i.e. the number of grid points taken in $[0, \pi]$ is 300 . The presented design examples are, in fact, related to the optimal design of twochannel IIR NDF banks with low group delay. The performance for each of the designed filter banks is evaluated in terms of the peak reconstruction error (PRE), the normalised peak stopband ripple (NPSR), and the stopband error energies (SEE) of the designed $H_{0}(z)$ and $H_{1}(z)$. They are given by

$$
\begin{aligned}
& \operatorname{PRE}(\mathrm{dB})=\max \left\{\left|20 \log _{10}\right| \hat{T}\left(\omega_{i}\right) \|\right\} \quad \text { for } \omega_{i} \in[0, \pi] \\
& \mathrm{NPSR}_{0}(\mathrm{~dB})=-20 \log _{10}\left(\max \frac{\left|H_{0}\left(\omega_{i}\right)\right|}{\sqrt{L L_{0}}}\right) \\
& \text { for } \omega_{i} \in\left[\omega_{s}, \pi\right], \quad \operatorname{SEE}_{0}=\sum_{\omega_{i} \in S_{3}}\left|H_{0}\left(\omega_{i}\right)\right|^{2} \\
& \mathrm{NPSR}_{1}(\mathrm{~dB})=-20 \log _{10}\left(\max \frac{\left|H_{1}\left(\omega_{i}\right)\right|}{\sqrt{L L_{1}}}\right) \\
& \text { for } \omega_{i} \in\left[0, \omega_{p}\right], \quad \mathrm{SEE}_{1}=\sum_{\omega_{i} \in S_{1}}\left|H_{1}\left(\omega_{i}\right)\right|^{2} .
\end{aligned}
$$

max. variation of $G D\left(\hat{T}\left(e^{j \omega_{i}}\right)\right)$

$$
=\max _{\omega_{i} \in[0, \pi]}\left|G D\left(\hat{T}\left(e^{j \omega_{i}}\right)\right)-k_{d}\right|(\text { samples })
$$

max. variation of passband $G D\left(H_{0}\left(e^{j \omega_{i}}\right)\right)$

$$
=\max _{\omega_{i} \in\left[0, \omega_{p}\right]}\left|G D\left(H_{0}\left(e^{j \omega_{i}}\right)\right)-k_{d} / 2\right|(\text { samples })
$$

max. variation of passband $G D\left(H_{1}\left(e^{j \omega_{i}}\right)\right)$

$$
=\max _{\omega_{i} \in\left[\omega_{s}, \pi\right]}\left|G D\left(H_{1}\left(e^{j \omega_{i}}\right)\right)-k_{d} / 2\right|(\text { samples })
$$

and max. variation of filter bank response

$$
=\max _{\omega_{i} \in[0, \pi]}\left|e^{-j \omega_{i} k_{d}}-\hat{T}\left(e^{j \omega_{i}}\right)\right|
$$

$G D(x)$ denotes the group delay of $x$.

For comparison, we also use the proposed technique for each design example with a FIR NDF bank and the same group delay $k_{d}$. For designing FIR NDF banks, the computations required for finding the denominators in the proposed iterative procedure are not necessary and can be discarded.

Example 1: We use the following design specifications: the group delay $k_{d}$ is $19 ; \omega_{p}=0.3 \pi$ and $\omega_{s}=0.5 \pi ; L_{0}=2$ and $L_{1}=3 ; M_{0}=N_{0}=10$ and $M_{1}=N_{1}=11$. In the FIR case, $H_{0}(z)$ and $H_{1}(z)$ have orders equal to 32 and 33 , respectively. Accordingly, the numbers of independent coefficients for the IIR and FIR NDF banks are 44 and 67, respectively. Moreover, the values of $\alpha, \beta, \gamma, \tau$, and $\epsilon$ are set to $0.012,0.012,0.05,0.5$, and $10^{-3}$, respectively, for the IIR NDF bank; they are set to $0.015,0.015,0.02,0.5$, and $10^{-3}$, respectively, for the FIR NDF bank.

Table 1 shows the significant design results for both of the designed IIR and FIR NDF banks. The resulting tap and reflection coefficients of the designed IIR NDF bank are

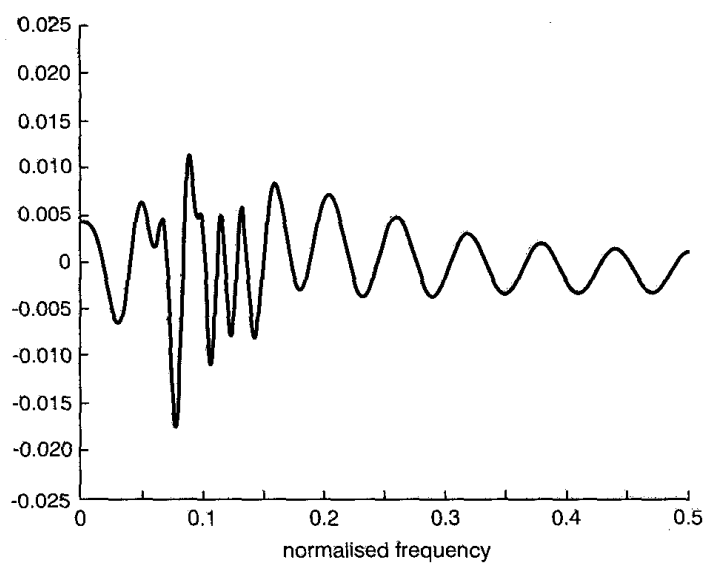

a

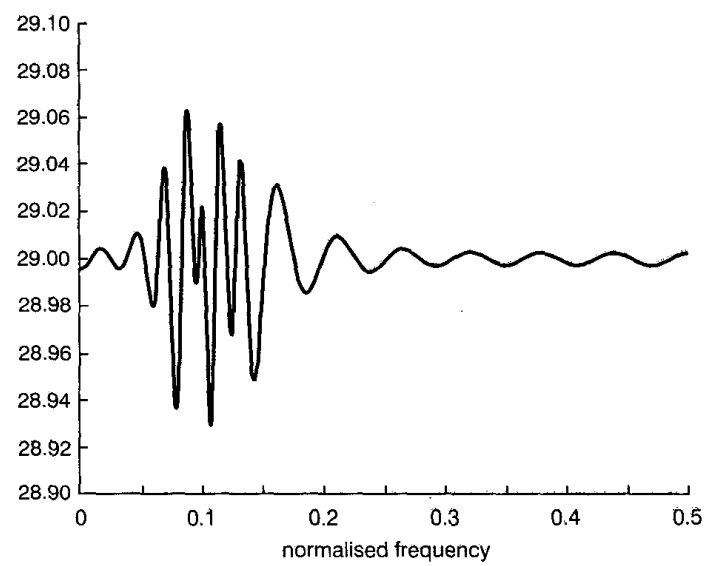

$b$

Fig. 8 Magnitude and group delay response of $\hat{T}\left(e^{j \omega}\right)$ for example 2 a Magnitude response $b$ Group delay response

listed in Table 2. Fig. 4 plots the corresponding magnitude responses and the group delays of $H_{0}\left(e^{j \omega \omega}\right) / \sqrt{ } L L_{0}$ and $H_{1}\left(e^{j \omega}\right) / \sqrt{ }\left(L L_{1}\right)$. Fig. 5 depicts the magnitude response and the group delay response in samples of $\hat{T}\left(e^{j \omega}\right)$ for the designed IIR NDF bank. The magnitude of $e^{-j^{k} d^{\omega}}-\hat{T}\left(e^{j \omega}\right)$

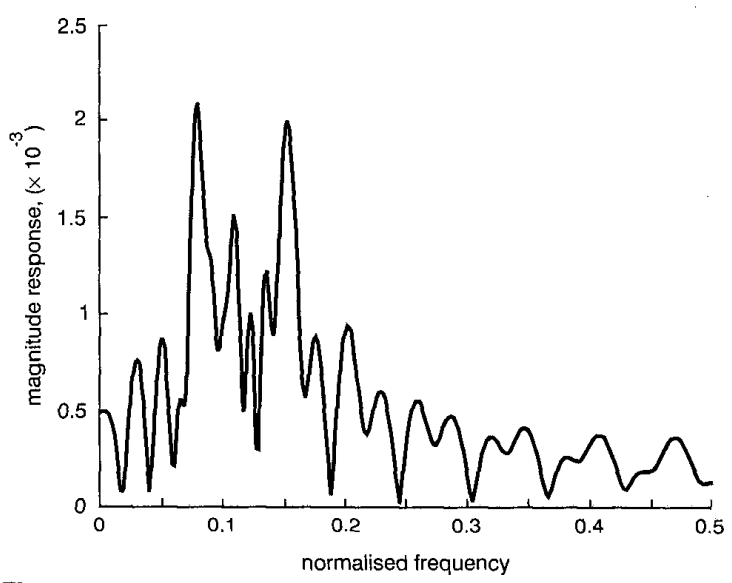

Fig. 9 Magnitude response of variation of filter bank response for example 2 
is shown in Fig. 6. From the design results of using the proposed technique, we note that the IIR NDF bank provides the advantage of requiring a lower order for achieving a comparable performance than the FIR NDF bank.

Example 2: The design specifications used are as follows: the group delay $k_{d}$ is $29 ; \omega_{p}=0.12 \pi$ and $\omega_{s}=0.28 \pi$; $L_{0}=1 \quad$ and $L_{1}=4 ; \quad M_{0}=13$ and $N_{0}=14 ; \quad$ and $M_{1}=N_{1}=17$. In the FIR case, both $H_{0}(z)$ and $H_{1}(z)$ have orders equal to 49 . Accordingly, the numbers of independent coefficients for the IIR and FIR NDF banks are 63 and 100 , respectively. Moreover, the values of $\alpha, \beta, \gamma, \tau$ and $\epsilon$ are set to $0.02,0.02,0.04,0.5$ and $5 \times 10^{-3}$, respectively, for the IIR NDF bank; they are set to $0.1,0.1,0.05,0.5$, and $10^{-3}$, respectively, for the FIR NDF bank.

Table 3 shows the significant design results for both of the designed IIR and FIR NDF banks. The resulting tap and reflection coefficients of the designed IIR NDF bank are listed in Table 4. Fig. 7 plots the corresponding magnitude responses and the group delays of $H_{0}\left(e^{j \omega}\right) /$ $\sqrt{ } L L_{0}$ and $H_{1}\left(e^{j \omega}\right) / \sqrt{ } L L_{1}$. Fig. 8 depicts the magnitude response and the group delay response in samples of $\hat{T}\left(e^{j \omega}\right)$ for the designed IIR NDF bank. The magnitude of $e^{-j^{k} d^{\omega}}-\hat{T}\left(e^{j \omega}\right)$ is shown in Fig. 9. We again observe from the design results that the IIR NDF bank provides the advantage of requiring a lower order for achieving a comparable performance than the FIR NDF bank.

\section{Conclusions}

We have employed recursive analysis filters with a lattice denominator for designing IIR NDF banks. At each iteration, the proposed design technique adjusts the tap and reflection coefficients for the analysis filters, to reduce the resulting squared error and keep the designed IIR analysis filters stable. For the first task, a linearisation scheme has been proposed to solve the resulting highly nonlinear programming problem. For the second task, the stability of the designed recursive analysis filters is ensured by incorporating an efficient stabilisation procedure. Computer simulations have shown the effectiveness of the proposed technique.

\section{Acknowledgments}

This work was supported by the National Science Council under Grant NSC88-2218-E002-031.

\section{References}

1 CROCHIERE, R.E.: 'Digital signal processor: sub-band coding', Bell Syst. Tech. J., 1981, 60, pp. 1633-1653

2 BELLANGER, M.G., and DAGUET, J.L.: 'TDM-FDM transmultiplexer: digital polyphase and FFT', IEEE Trans. Commun., 1974, 22, pp. 1199-1204

3 VARY, P., and HEUTE, U.: 'A short-time spectrum analyzer with polyphase network and DFT', Signal Process., 1980, 2, pp. 55-65

4 WOODS, J.W., and O'NEIL, S.D.: 'Subband coding of images', IEEE Trans. Acoust. Speech Signal Process. 1986, 34, pp. 1278-1288

5 NAYEBI, K., BARNWELL, T.P., and SMITH, M JT. 'Nonuniform filter banks: a reconstruction and design theory', IEEE Trans. Signal Process., 1993, 41, pp. $1114-1127$

6 WADA, S.: 'Design of nonuniform division multirate FIR filter banks', IEEE Trans. Circuits Systems II, Analog Digit. Signal Process., 1995, 42, pp. 115-121

7 LEE, J.-H., and HUANG, S.-C.: 'Design of two-channel nonuniformdivision maximally decimated filter banks using $L_{1}$ criteria', IEEE Proc. Vis. Image Signal Process., 1996, 143, pp. 79-83

8 LEE, J.-H., and TANG, D.-C.: 'Optimal design of two-channel nonuniform-division FIR filter banks with $-1,0$, and +1 coefficients', IEEE Trans. Signal Process., 1999, 47, pp. 422-432

9 EKANAYAKE, M.M., and PREMARATNE, K.: 'Two-channel IIR QMF banks with approximately linear-phase analysis and synthesis filters', IEEE Trans. Signal Process., 1995, 43, pp. 2313-2322

10 TAY D.B.H. 'Design of causal stable IIR perfect reconstruction filter banks using transformation of variables', IEE Proc., Vis. Image Signal Process., 1998,145, pp. $287-292$

11 OKUDA, M., FUKUOKA, T., IKEHARA, M., and TAKAHASHI, S.-I. 'The design of two-channel perfect reconstruction IIR filter banks with causality', Electron. Commun. Jpn., 1998, 81, pp. 22-31

12 LIM, Y.C.: 'On the synthesis of IIR digital filters derived from AR lattice network', IEEE Trans. Acoust. Speech Signal Process., 1984, 32, pp. $741-749$

13 BELICZYNSKI, B., KALE, I., and CAIN, G.D.: 'Approximation of FIR by IIR digital filters: an algorithm based on balanced model reduction', IEEE Trans. Signal Process., 1992, 40, pp. 532-542

14 OPPENHEIM, A.V., and SCHAFER, R.W.: 'Discrete-time signal processing' (Prentice-Hall, Englewood Cliffs, New Jersey, 1989)

15 BURRUS, C.S., BARRETO, J.A., and SELESNIK, I.W.: 'Iterative reweighted least-squares design of FIR filters', IEEE Trans. Signal Process., 1994, 42, pp. 2926-2936

16 NELDER, JA., and MEADE, R. "A simplex method for function minimization', Comput. J., 1965, 7, pp. 308-313 